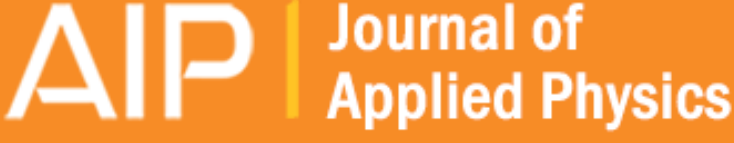

\section{A biodetection method using magnetic particles and micro traps}

Fuquan Li, loanna Giouroudi, and Jürgen Kosel

Citation: Journal of Applied Physics 111, 07B328 (2012); doi: 10.1063/1.3678304

View online: http://dx.doi.org/10.1063/1.3678304

View Table of Contents: http://scitation.aip.org/content/aip/journal/jap/111/7? ver=pdfcov

Published by the AIP Publishing

\section{Articles you may be interested in}

Progress in Using Magnetic Nanoobjects for Biomedical Diagnostics

AIP Conf. Proc. 1025, 28 (2008); 10.1063/1.2956815

Moment Selective Digital Detection of Single Magnetic Beads for Multiplexed Bioassays

AIP Conf. Proc. 1025, 176 (2008); 10.1063/1.2956812

Phase sensitive enhancement for biochemical detection using rotating paramagnetic particle chains

J. Appl. Phys. 96, 6831 (2004); 10.1063/1.1809269

Biodetection using magnetically labeled biomolecules and arrays of spin valve sensors (invited)

J. Appl. Phys. 93, 7281 (2003); 10.1063/1.1544449

Detection of single micron-sized magnetic bead and magnetic nanoparticles using spin valve sensors for biological applications

J. Appl. Phys. 93, 7557 (2003); 10.1063/1.1540176

MIT LINCOLN

LABORATORY CAREERS

Discover the satisfaction of innovation and service

to the nation
- Space Control

- Air \& Missile Defense

- Communications Systems \& Cyber Security

- Intelligence, Surveillance and

Reconnaissance Systems

- Advanced
Electronics
- Tactical Systems
- Homeland
Protection
- Air Traffic Control

LINCOLN LABORATORY

MassachusetTs Institute of TeChNOLOgY

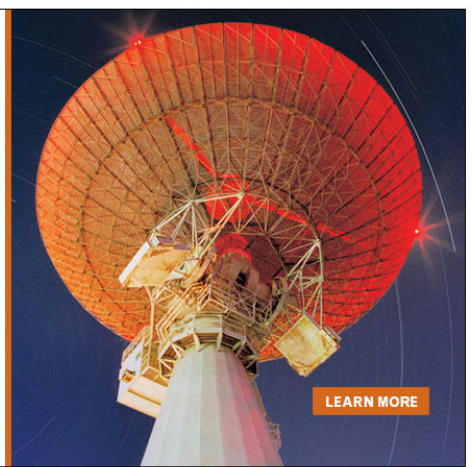




\title{
A biodetection method using magnetic particles and micro traps
}

\author{
Fuquan $\mathrm{Li}^{1}{ }^{1, \mathrm{a})}$ loanna Giouroudi, ${ }^{2}$ and Jürgen $\mathrm{Kosel}^{1}$ \\ ${ }^{1}$ Division of Physical Sciences and Engineering, King Abdullah University of Science and Technology, \\ Thuwal 23955-6900, Kingdom of Saudi Arabia \\ ${ }^{2}$ Institute of Sensor and Actuator Systems, Vienna University of Technology, 1040 Vienna, Austria
}

(Presented 3 November 2011; received 23 September 2011; accepted 28 November 2011; published online 9 March 2012)

\begin{abstract}
The general working principle of magnetoresistive sensors for biological applications is to specifically attach bioanalytesto magnetic particles and then detect the particles that are immobilized on the sensor surface. The immobilization of the particles on the sensor surface commonly uses biomolecular interactions, e.g., antigen-antibody. Thus, the sensor surface needs to be functionalized via biological treatments in order to capture certain bioanalytes. In the presented work, a new method is proposed, which does not rely on functionalization of the sensor surface. Current carrying microstructures in combination with mechanical micro traps are used to immobilize magnetic particles. Analyte detection is based on the difference in size between bare magnetic particles and particles with analyte attached, which causes a different number of particles to be captured in the micro traps. (C) 2012 American Institute of Physics. [doi:10.1063/1.3678304]
\end{abstract}

\section{INTRODUCTION}

Micro devices employing superparamagnetic particles and magnetic micro sensors have attracted significant attention in recent years, especially for biological and medical applications. They take advantage of the facts that superparamagnetic particles can be manipulated by permanent magnets ${ }^{1,2}$ or current carrying magnetic microstructures ${ }^{3}$ and sensed by integrated magnetic sensors. ${ }^{3-5}$ Additionally, they can be made biocompatible and functionalized to target specific bioanalytes.

Magnetic biosensors generally employ a "sandwich" approach to immobilize bioanalytes. ${ }^{6}$ First of all, one microbiological substance, the "probe," is immobilized on top of a magnetic sensor. Secondly, the bioanalyte, also called the "target," passes by the probes. Targets and probes bind specifically, via DNA-DNA, antigen-antibody, or ligandreceptor interaction. Finally, magnetic particles, which can react with targets, are introduced to the sensor's surface to let them bind with the targets. Such a "sandwich" approach is now commonly used in magnetic biosensor devices. ${ }^{4-8}$ The biggest disadvantage of this type of biosensors, is that they are prone to time-dependent changes of the functionalization layer such as aging and contamination. Long-term stability is therefore an issue with those types of biosensors. It is also difficult to control or acquire the amount of immobilized probes.

In the presented work, a method is proposed, which does not rely on functionalization of the sensor's surface.

\section{METHOD}

Instead of using biological interactions to immobilize magnetic particles and targets, a gold microstructure and a mechanical trapping chamber are used. The trapping cham-

\footnotetext{
a) Author to whom correspondence should be addressed. Electronic mail: fuquan.li@kaust.edu.sa.
}

ber is located above the gold microstructure (Fig. 1), which carries a current, creating a non-uniform magnetic field. Magnetic particles in the vicinity of the microstructures are attracted into the trapping chamber.

If a sample solution contains only superparamagnetic particles (SMPs) but no targets, the number of particles that are trapped inside the chamber will be larger than in case the target is present in the sample solution. This is due to the increase in size as the targets bind to SMPs, which will cause the chamber to fill up with a smaller number of particles. Therefore, the difference in volume between the bare SMPs and the compound of SMPs and the target in combination with the trap is the basis for the proposed detection method. In order to maintain a high sensitivity, the size of the target analyte and the magnetic particle should be in the same range.

The design of the device plays a crucial role with respect to the function and sensitivity. To maintain high sensitivity for a system using a sensor underneath the trap, the particles should be kept as close to the bottom of the trap as possible. Therefore, it is better to just trap a single layer of magnetic particles, which can be controlled by the size of the trapping chamber or the current through the gold microstructures. In order to be able to trap particles from a flowing liquid, the magnetic force needs to be larger than the hydrodynamic drag force. The magnetic force is basically determined by the current through the gold microstructure, and the drag force is determined by the flow velocity and viscosity of the liquid.

The magnetic force of a current carrying wire on magnetic particles in fluids is given by ${ }^{6}$

$$
F_{\text {mag }}=\frac{v_{\chi}}{\mu_{o}}(B \cdot \nabla) B,
$$

$\chi$ is the susceptibility of the magnetic particle, $V$ is the volume of the magnetic material in the particles, and $B$ is the magnetic flux density. 


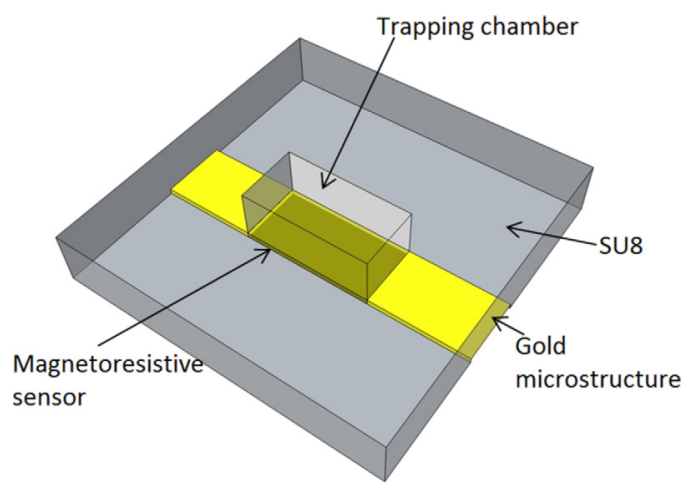

FIG. 1. (Color online) Schematic of the device consisting of a gold microstructure to capture magnetic particles in a trapping chamber.

The hydrodynamic drag force is caused by the resistance of the molecules of the fluid and is proportional to the viscosity of the fluid. The expression for the hydrodynamic drag force is

$$
F_{d}=3 \pi D \eta v
$$

$D$ is the diameter of the magnetic particle, $\eta$ is the viscosity of the fluid, and $v$ is the flow velocity.

In order for magnetic particles to be trapped by the magnetic force, the equation $F_{m a g}=F_{d}$ can be used to obtain the maximum velocity of a flowing fluid from which the magnetic particles can still be trapped.

Simulations of magnetic field and force distribution were carried out with commercial finite-element software (COMSOL) using the following parameters: The width and height of the gold structure are $6 \mu \mathrm{m}$ and $0.5 \mu \mathrm{m}$, respectively, and the height of the trapping chamber (made from SU8) is $5 \mu \mathrm{m}$ (Fig. 2). The current flowing in the gold structure is $30 \mathrm{~mA}$. The parameters of the magnetic particle are those of Dynabeads ${ }^{\circledR} \mathrm{M}-270$, which has a diameter of $2.8 \mu \mathrm{m}$ and a magnetic susceptibility of $0.17 .^{9}$ The magnitude of the magnetic flux density is shown in Fig. 2. The highest field values occur at the edges of the gold structure and the field strength rapidly decreases toward the top of the trap.

The lateral magnetic force is the most important one, since it determines whether a magnetic particle can be attracted to the chamber. Assuming a flow velocity of $1 \mu \mathrm{m} / \mathrm{s}$,

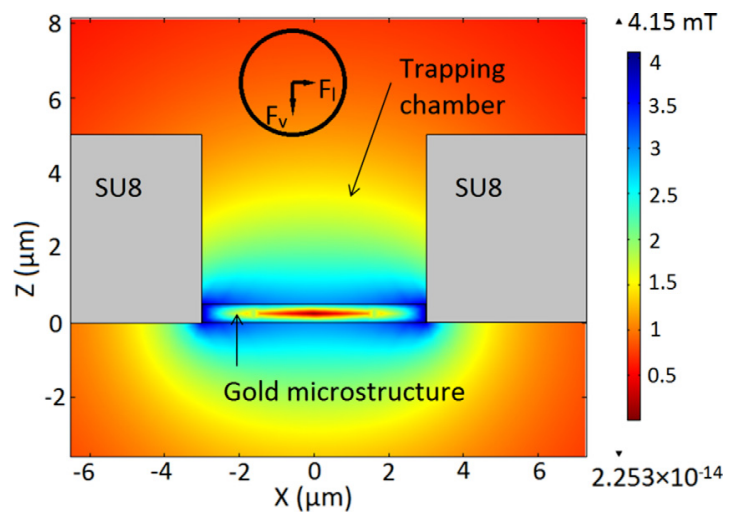

FIG. 2. (Color online) Magnitude of magnetic flux density and schematic cross-section of the device.

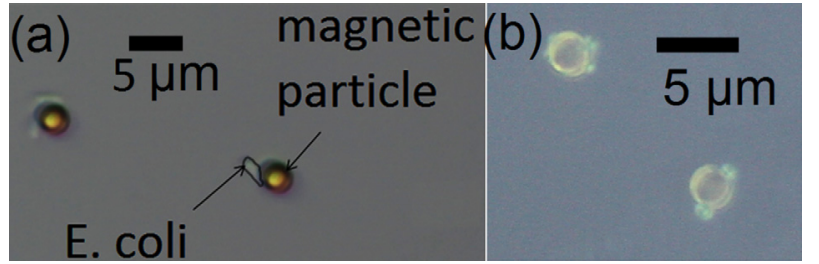

FIG. 3. (Color online) (a) Streptavidin coated superparamagnetic particles bound to E. coli bacteria, (b) Streptavidin coated superparamagnetic particles bound to fluorescent particles via Bovine serum albumin (BSA). Note, the outline of one bacterium is enhanced by black lines.

a particle in water at $25^{\circ} \mathrm{C}$ can be attracted from a distance of $11.5 \mu \mathrm{m}$. The efficiency of this trapping mechanism could be enhanced, e.g., by employing an external permanent magnet or an integrated particle concentration system. ${ }^{3}$

\section{EXPERIMENTS AND DISCUSSION}

\section{A. Device fabrication}

The device was fabricated on top of a silicon wafer covered with $100 \mathrm{~nm} \mathrm{Si}_{3} \mathrm{~N}_{4}$. The wafer was first covered with a $20 \mathrm{~nm}$ thick titanium film to enhance the adhesion between $\mathrm{Si}_{3} \mathrm{~N}_{4}$ and the $500 \mathrm{~nm}$ thick gold film deposited on top of it. After deposition and patterning of a photoresist layer, the gold film was dry etched. Then, the trapping chamber was fabricated on top of the Au wire applying a $5 \mu \mathrm{m}$ thick layer of SU8, followed by patterning the trapping hole.

\section{B. Trapping experiments}

Trapping experiments were carried out using superparamagnetic Dynabeads ${ }^{\circledR}$ M-270 particles with a diameter of $2.8 \mu \mathrm{m}$ and a magnetic susceptibility of 0.17 . Two different kinds of target analytes were tested: $E$. coli bacteria and Bovine Serum Albumin (BSA) protein (Fig. 3(a) and Fig. 3(b)). E. coli $\mathrm{K} 12$ bacteria were attached to the particles via streptavidin and anti-E. coli antibodies(ab20640). BSA was attached to the particles via a streptavidin-biotin link. However, since the size of BSA is much smaller than the size of the Dynabeads, the sensitivity of the method would be very low. Therefore, fluorescent particles (Fluoresbrite ${ }^{\circledR}$ YG Carboxylate Microspheres) of $1.00 \mu \mathrm{m}$ were attached to BSA. The carboxyl groups $(\mathrm{COOH})$ on the fluorescent particles is activated with carbodiimide to create an active ester that can couple with primary amines $\left(\mathrm{NH}_{2}\right)$ on BSA. Using this

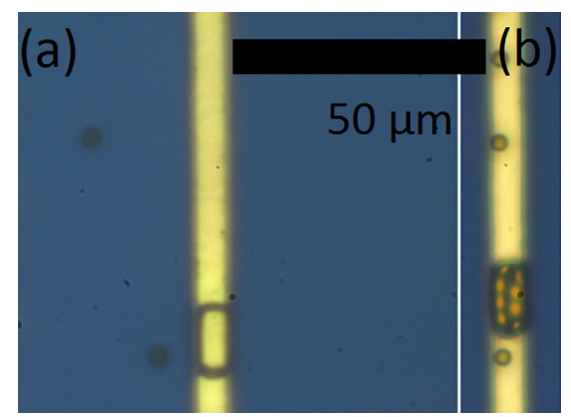

FIG. 4. (Color online) (a) the trap and superparamagnetic particles, (b) 8 magnetic particles trapped inside the chamber. 


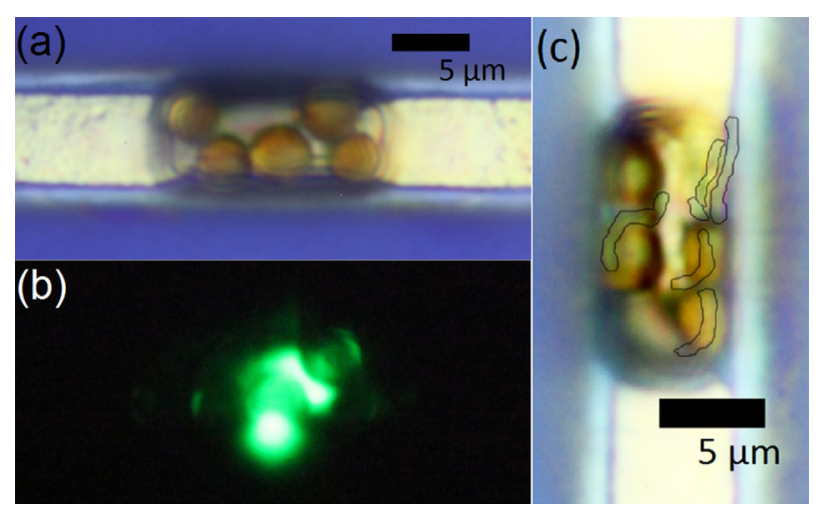

FIG. 5. (Color online) (a) 5 magnetic particles with BSA and fluorescent particles trapped, (b) fluorescent particles, (c) 4 magnetic particles with E. coli trapped. Note, the outline of the bacteria is enhanced by black lines.

approach, small target analyte can still be detected with rather large magnetic particles without compromising sensitivity.

Three experiments were performed in which droplets of solution containing only magnetic particles, magnetic particles with E. coli and magnetic particles with BSA were placed on top of the device. Between each experiment, the device was thoroughly rinsed with de-ionized water. Before applying electric current, the particles passed the trapping structure without being influenced and the trap remained empty (Fig. 4(a)). When current was applied, the particles were attracted and eventually filled the trap. Figure 4(b) shows the trap being entirely filled with 8 magnetic particles. When the experiment was repeated with the solutions containing the analytes, the trap was filled with only 5 particles in case of BSA (Fig. 5(a) and Fig. 5(b)) and 4 particles in case of E. coli bacteria (Fig. 5(c)). Therefore, the number of magnetic particles has changed by $50 \%$ and $38 \%$ for E. coli and BSA, respectively.

\section{CONCLUSION}

A new method has been demonstrated to enable discrimination of bare magnetic particles from magnetic particles with target analytes attached to them. The method does not require functionalization of a sensor surface and is based on the change in volume of the bare particles and particlesample compound. Magnetic forces produced by a current carrying microstructure are utilized to attract particles into a mechanical trap. In combination with a sensing device, e.g., a magnetic micro sensor underneath the trap, this method can be used to detect the presence of a target sample in a liquid. The new method can be applied for different kinds of bioanalytes that can be immobilized on the surface of magnetic particles. In addition, it can easily be integrated with sensors for analyte detection and could greatly enhance the reliability of magnetic biosensor.

${ }^{1}$ G. P. Hatch and R. E. Stelter, J. Magn. Magn. Mater. 225, 262 (2001).

${ }^{2}$ N. Pamme and A. Manz, Anal. Chem. 76, 7250 (2004).

${ }^{3}$ C. P. Gooneratne, I. Giouroudi, C. Liang, and J. Kosel, J. Appl. Phys. 109, 07E517 (2011).

${ }^{4}$ D. R. Baselt, G. U. Lee, M. Natesan, S. W. Metzger, P. E. Sheehan, and R. J. Colton, Biosens. Bioelectron. 13, 731 (1998).

${ }^{5}$ J. Rife, Sens. Actuators A 107, 209 (2003).

${ }^{6}$ M. A. M. Gijs, Microfluidics and Nanofluidics. 1, 22, (2004).

${ }^{7}$ R. L. Millen, T. Kawaguchi, M. C. Granger, and M. D. Porter, Anal. Chem. 77, 6581 (2005).

${ }^{8}$ W. Shen, B. D. Schrag, M. J. Carter, J. Xie, C. Xu, S. Sun, and G. Xiao, J. Appl. Phys. 103, 07A306 (2008).

${ }^{9}$ P. Tierno, T. Johansen, and T. Fischer, Phys. Rev. Lett. 99, 038303 (2007). 